\title{
Mutli-resolution Cloth Simulation based on Particle Position Correction
}

\author{
Sabrina Benameur \\ LESIA Laboratory \\ Biskra University \\ Biskra, Algeria
}

\author{
Nour Eddine Djedi \\ LESIA Laboratory \\ Biskra University \\ Biskra, Algeria
}

\begin{abstract}
This paper presents a multi-resolution cloth simulation based on first order finite element method. Previous works on multi-resolution cloth simulation used discrete cloth models like mass-spring in combination with one of subdivision schemes. The major drawback of such models is the difficulty of adjusting their parameters, which slow down the convergence of the model, especially when using different levels of details. Models based on first order finite element method can handle arbitrary triangle meshes, not necessarily regular ones. In order to contribute to solve the problem of the divergence of simulations, a method for correcting the positions of particles is proposed. And to improve the simulation, criteria such as the angle of subdivision, the shape of the fabric and the number of the level of detail are taken into account.
\end{abstract}

\section{Keywords}

Cloth Simulation, Particle Systems, Finite Elements, Multiresolution, Level of Details (LODs), Position correction.

\section{INTRODUCTION}

Cloth simulation was based on the majority of work on massspring systems (for surveys see [1, 2]). These models represent the behavior of the cloth by forces connecting the masses representing the cloth surface at the distance separating them from their neighbors. They became popular thanks to the simplicity of their formulation, which facilitates in particular the use of implicit integration methods [3, 4, 5]. Unfortunately, mass-spring systems are unable to model surface elasticity accurately, and they are particularly inaccurate for anisotropic and non-linear models like cloth.

Recently, the first order finite elements were introduced to obtain the best compromise between precision and computing speed. They were expressed like particle systems, of which all the numerical integration techniques developed in this context were used. However, some adaptations are necessary to be able to correctly manage the context of large deformations due to the cloth simulation.

A new approach corotational was proposed and became very widespread because it combines the simplicity of calculations by using the linear tensor of Cauchy with large deformations. This method was used successfully by Etzmuss et al. [6] for cloth simulation. It is essentially adapted to simulate linear isotropic materials with large deformations. Besides significant additional computation, these corotational schemes make it complicated to express anisotropic mechanical behaviors, particularly if they are nonlinear. Attempts to avoid the corotational formulation [7] also lead to inaccurate approximations of the shear strain and stress for large deformations. In order to preserve accurately the simulation, a curvature model is added by using the schemes defined by [ 8].

In a recent work, Volino et al. [9] have presented a simple and accurate model which offers a highly accurate representation of cloth deformation based on continuum mechanics. The presented scheme remains accurate for large deformations without the need of complicating the computation process with additional transformations, such as co-rotational formulations. In addition, it can be expressed as a classical Particle System (with particle forces directly computed from particle positions). The exact Jacobian proposed in this model can also be efficiently computed, offering efficient and stable simulations through the use of implicit integration methods (Inverse Euler, BDF-2, etc.). But despite that the implicit integration methods ensure the stability of the model, but it requires additional computations. The authors in [9] mentioned that it is possible to use their model with one of explicit integration methods, but, to ensure the stability of the model it is necessary to use small time steps.

In this work, particle system model is used based on continuum mechanics with first order Finite Element method. The model is based on the scheme proposed by Volino et al. [9]. However, in this case an explicit integration method has been used. The garment is simulated by irregular triangular meshes. In order to improve the simulation different parameters are used such as the wind, gravity and friction. Multi-resolution is exploited in the model by using the polyhedral subdivision scheme. Therefore, this scheme induces locally a great number of similar triangles, thus makes it possible to keep the original form of the grid. In addition, this scheme is characterized by a simple process and of low complexity.

With the explicit integration method the stability of simulation cannot be obtained only if a very small time step is used. The contribution in this work relates to the resolution of the problem of the divergence, by proposing a method based on particle position correction. Thus the displacement of each particle at each iteration is limited, so that each particle cannot move more than a certain distance (threshold) which is determined after several tests (see section 4).

Since the computation time also depends on the resolution of the used grid, which determines the smoothness of the details of the garments (folds). A too fine resolution is likely to increase the computation time, whereas a simplified grid leads to a loss of details. Distribution of grid nodes on cloth surface according to the level of detail could then reduce the cost of calculating appreciably. Thus, it was necessary to use an adaptive grid in order to ensure the convergence of the model. 
Basing on this principle, several researchers exploited multiresolution representation in cloth and garment simulation to improve the computation times.

\subsection{Related works}

In this section an overview about the most important works in the area of multi-resolution cloth simulation is given.

Hutchinson et al. represented in [10] cloth by a mass-spring system with a rectangular grid. The multi-resolution was introduced by locally adding masses and springs to refine the system when the angle between two neighboring springs exceeds a certain tolerance value. The objective was the resolution of the problem of static mass-spring systems (of which the resolution of the grid does not change during the time).

Within the same order of idea, Burgy [11] has developed the same system by proposing the representation of mass-spring systems with a triangular grid, instead of a rectangular grid witch considered as restrictive. Except that the swing between the levels of details is done only in one direction. Therefore, the mass-spring system becomes static after some iterations and the mesh becomes uniform, causing so the loss of the concept of multi-resolution. In order to solve this problem and basing on the model of Burgy [11], we have proposed in [12] a method of cancellation of subdivision in the zones sufficiently flat, this enabled us to animate pieces of cloth and to make a swing between the different levels of details in the two directions. A method of refinement of a rectangular grid was proposed in [13] for adapting the cloth on the shape of the object during the simulation process. In order to generate folds, the adaptive scheme consists of refining locally the grid when the curvature exceeds a given threshold.

In [14], F. Birra and M. Santos have presented a technique that simulates cloth surfaces with adaptive levels of details without degrading the physical surface properties or the stability of the simulation. The underlying mesh operations are derived from subdivision surfaces, but the refinement criterion is adapted in order to prevent the numerical simulation from diverging.

Lee et al. [15] have used a combination between a massspring system and Loop subdivision scheme for refining a triangular grid. The subdivision steps are pre-computed in order to get a multi-resolution hierarchy. This is used to adaptively reduce the dimension of the linear system which must be solved by an implicit integration step.

In the context of garment simulation, Li and Volkov [16] presented an adaptive version of Baraff's cloth simulation method [17] which is able to handle arbitrary triangle meshes. An ascending approach is adopted for the refinement of the grid, which does not require the pre-computation and the storage of multi-resolution hierarchy. Levels of detail are built during the simulation, which allow the local simplification and subdivision.

In [18] Thanh proposed a novel garment simulation method, which relates to the design of a powerful automatic preposition of garment around a mannequin, in order to reduce the computation times. The idea was firstly, to discretize garment in various resolutions. Thus, a minimization of system resolution is applied iteratively in a very short time.

In these cases, system stability based on mass-spring model depends on system parameters such as masses of the points, stiffness's of the springs, the speed of the wind,...
Nevertheless, these parameters which are difficult to fix, constitute the main issue for this model. In contrast to these previous works that use mass-spring systems which are not always convergent, our model is based on continuum mechanics

In the last years, different authors have proposed to use a continuous model to simulate cloth with or without multiresolution. Etzmuss et al. [19] have used a finite difference discretization of the model in order to solve the differential equations. This discretization has only enabled him to handle quadrilateral meshes. In a second work [6], they have presented an efficient approach based on the finite element method with a co-rotating formulation which can handle arbitrary triangle meshes. Volino et al. presented in [9] a cloth simulation system based on continuum mechanics which is able to simulate nonlinear anisotropic materials. They demonstrated that the use of a simplified continuous representation allows obtaining a good compromise between precision and computing speed. In this work, the movement of the garment is implicitly related to the movement of the body that wears it

Thereafter, Multi-resolution was introduced into continuous models in different work; Brochu et al. [20] used the model suggested by Etzmuss et al. [19] to demonstrate that their method of continuous collision detection was able to handle adaptive meshes.

J. Bende et al. proposed in [21] an adaptive cloth model based on continuum mechanics. They used the linear finite element method (FEM) in combination with a co-rotating formulation to have an efficient simulation. Their method works with triangular elements which are defined by the adaptive triangular mesh of their cloth model. The resolution of the grid is changed during simulation by using the $\sqrt{3}$ refinement scheme. This scheme allows the subdivision of an irregular triangular grid while preserving the quality of the simulation.

In a recent work, J. Bender et al. [22] exploited multiresolution in a model based on the shape matching approach. They presented a modification of the original shape matching method to preserve the folds of the garment. They also demonstrated that their method could be handled efficiently in the GPU

\subsection{Overview}

A short overview of multi-resolution garment simulation method is given in this section. The details of each step will be presented in the following paragraphs

In the simulation a triangular mesh of particles is used as cloth model. Each particle has a mass $\mathrm{m}$, a position $\mathrm{P}$ and a speed V.

A simulation step for this cloth model is performed as follows:

1. Perform a simulation step with the continuous model to determine the forces acting on each particle and to get the new velocity and position of each particle (see section 2.1)

2. Correct particle positions (see section 4) in order to improve the animation and to exploit the multiresolution.

3. Perform collision detection between cloth and some primitive and resolve all collisions (see section 2.2) 
4. Simulate cloth model using a multi-resolution method (see section 3)

\section{CLOTH SIMULATION WITH FINITE ELEMENT METHOD}

\subsection{Cloth model}

Cloth is the physical result of the interlacing of textile wire warp "longitudinal direction" and weft "transverse direction". The mode of interlacing of the two series of wire, by taking account of their nature, their mechanical, physical and chemical properties, plays a role in the aesthetic effect and influences largely the quality of cloth. The regular textile wire interlacing warp and weft form flexible surface known as cloth. This one is discontinuous, anisotropic, with nonlinear elasticity and prone to hysteresis [23].

This work proposes a model based on continuum mechanics. In which cloth and garment are presented with an irregular triangular grid. For that the first order finite element method was chosen to solve the movement equation. The simulation method is based on the model of Pascal Volino presented in [9].

In this model [9], it is shown that the use of a simplified continuous representation like the first order finite element methods makes it possible to obtain a good compromise between precision and computing speed. This model consists in representing the cloth by a particle system, where each triangle of the grid represents an element. Each element is treated separately, and then performs an assembly which satisfies the equilibrium of the forces in each vertex. Indeed, this model permits to express explicitly and precisely the stress and strain state in the surface of a triangle according to the positions and the forces on its vertices.
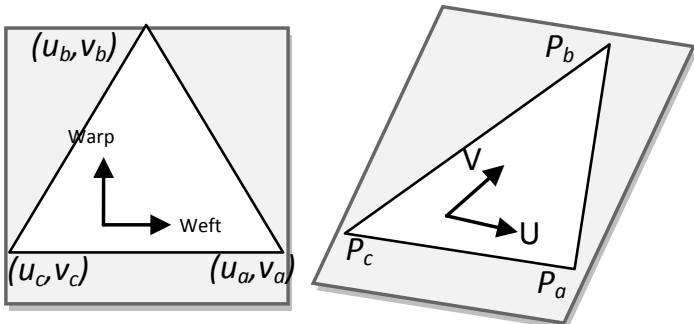

Figure: $1 \mathrm{~A}$ triangle element of the initial state is defined in 2D parametric coordinates (left), and deformed in 3D world coordinates (right) [9].

The goal is to compute the deformation state of a triangle element directly from the positions of its vertices. Each element (triangle) is described by its 2D parametric coordinates (ua,va),(ub,vb),(uc,vc) of its vertices and by the $3 \mathrm{D}$ world coordinates $\mathrm{Pa}, \mathrm{Pb}, \mathrm{Pc}$ of its vertices after its deformation. The weft and warp vectors are expressed in 3D world coordinates as $\mathrm{U}$ and $\mathrm{V}$, they will be used to measure the deformation state of an element.

At the beginning, in a Pre-computation step the vertex distribution factors are evaluated and stored as follows [9]:

$\begin{cases}r_{u a}=d^{-1}\left(v_{b}-v_{c}\right) & r_{v a}=d^{-1}\left(u_{c}-u_{b}\right) \\ r_{u b}=d^{-1}\left(v_{c}-v_{a}\right) & r_{v b}=d^{-1}\left(u_{a}-u_{c}\right) \\ r_{u c}=d^{-1}\left(v_{a}-v_{b}\right) & r_{v c}=d^{-1}\left(u_{b}-u_{a}\right)\end{cases}$

$d=u_{a}\left(v_{b}-v_{c}\right)+u_{b}\left(v_{c}-v_{a}\right)+u_{c}\left(v_{a}-v_{b}\right)$

During simulation, at each iteration the forces applied to the particles must be calculated to determine their corresponding velocities and positions. For that and according to the work suggested in [9] the following characteristics should be calculated for each element of cloth:

- Vectors $\mathrm{U}$ and $\mathrm{V}$ by the following equation:

$$
U=\sum_{i \in(a, b, c)} r_{u i} P_{i} \quad V=\sum_{i \in(a, b, c)} r_{v i} P_{i}
$$

In this case, each vector $\mathrm{U}$ and $\mathrm{V}$ correspond to the current sum of vertices positions of the triangle. These positions are weighted by the distribution factors calculated using the formula (1) given previously Strain values $\varepsilon_{\mathrm{uu}}, \varepsilon_{\mathrm{vv}}, \varepsilon_{\mathrm{uv}}$ by the equation (4) following:

$$
\left\{\begin{array}{c}
\varepsilon_{u u}=\frac{1}{2}\left(U^{T} U-1\right) \\
\varepsilon_{v v}=\frac{1}{2}\left(V^{T} V-1\right) \\
\varepsilon_{u v}=\frac{1}{2}\left(U^{T} V+V^{T} U\right)
\end{array}\right.
$$

Stress values $\sigma_{\mathrm{uu}}, \sigma_{\mathrm{vv}}, \sigma_{\mathrm{uv}}$ by the equation (5) below, by using the mechanical behavior of the material, in this work only the property of elasticity has been considered.

$$
\left\lceil\begin{array}{c}
\sigma_{u u} \\
\sigma_{v v} \\
\sigma_{u v}
\end{array}\right\rceil=E\left[\begin{array}{c}
\varepsilon_{u u} \\
\varepsilon_{v v} \\
\varepsilon_{u v}
\end{array}\right\rceil
$$

In this case, the symmetric matrices $\mathrm{E}$ represent the elastic stiffness of the material.

From the preceding equations, vertices forces of the current triangle $\mathrm{Fa}, \mathrm{Fb}, \mathrm{Fc}$ can be determined according to the following equation:

$$
F_{j}=-\frac{|d|}{2}\left(\sigma_{u u}\left(r_{u j} U\right)+\sigma_{v v}\left(r_{v j} V\right)+\sigma_{u v}\left(r_{u j} V+r_{v j} U\right)\right)
$$

A more realistic simulation of garment movement requires the explicit addition of parameters such as: wind, gravity and friction. These parameters are modelled by external forces noted respectively Fv, Fgr, Ffr.

The gravitation Fgr is a force simple to represent, directed downwards and proportional to the mass. The force of friction $\mathrm{F}_{\mathrm{fr}}$ is often introduced to give certain stability to simulation. It acts directly on the opposite sense of particle velocity by the following equation:

$$
\overrightarrow{F_{f r}}=-C \overrightarrow{V_{i}}
$$

Where $\mathrm{C}$ is the viscosity coefficient of the milieu.

The effect of the wind on the cloth is calculated on each triangle individually. The wind force $F_{v}$ applied to each particle will be thus the sum of the force of the wind applied to its surrounding triangles. At each iteration, this force must be calculated by:

$$
F_{v}=N *\left(\|N\| \cdot V_{v}\right)
$$

Where $\mathrm{N}$ is the normal of the triangle having the norm $\mathrm{V}_{\mathrm{v}}$ : the wind velocity.

To determine the new positions and velocities of the particles, any integration method can be used. Several methods are used in the literature, explicit methods are very simple and easy to implement, but they require very small time step to ensure convergence of the system [7]. Implicit methods have proved their convergence, but they differ from their complexity, their accuracy and their stability. The majority of these methods 
have shown that the use of the Jacobian and its accurate evaluation are necessary for good convergence and stability of simulation systems [7, 9, 17]. But the evaluation of the Jacobian is complicated in terms of understanding and calculating of its formula. This work proposes to use the modified Euler method (9) (10). In order to resolve the problem of the divergence, a method based on particle position correction is proposed (see section 4).

$$
\begin{gathered}
V_{i}^{n+1}=V_{i}^{n}+h \frac{F_{i}^{n}}{2 * m_{i}} \\
P_{i}^{n+1}=P_{i}^{n}+h V_{i}^{n+1}
\end{gathered}
$$

This method uses the time step $\mathrm{h}$ with $\mathrm{F}_{\mathrm{i}}^{\mathrm{n}}$ that represents the force of particle $i$ and $V_{i}^{n}$ and $V_{i}^{n+1}$ respectively $P_{i}^{n}$ and $\mathrm{P}_{\mathrm{i}}^{\mathrm{n}+1}$ which represent velocities, respectively, positions of particle $\mathrm{i}$ in iterations $\mathrm{n}$ and $\mathrm{n}+1$.

\subsection{Collision handling}

Let us point out that this problem relates initially, to the collision detection, i.e. the geometrical contact between two complex surfaces and in second place, the determination of the response to these collisions. In other words, how this geometrical contact can affect the cloth, and how this change is integrated effectively into simulation [24].

In this work the problem of cloth collision is handled with simple primitives like the sphere and the cylinder. The principle of collision handling with these objects is simpler in this case. Indeed, at each iteration, a test is performed to see if the particle is inside the object. In the case of the sphere, for example, the test is to check whether the distance between the position of the particle and its center is lower than its radius. If it is the case, then there is collision and it is thus necessary to move the particle to the nearest point on the surface of the sphere.

\section{MULTI-RESOLUTION GARMENT SIMULATION}

The choice of the mesh resolution, which represents cloth/garment, is important in simulation. Indeed, too refined mesh risks increasing the computation times, while too coarse mesh will give inadequate results. The representation of the mesh by various levels of details can solve this problem. Since the mesh resolution is refined in the parts requiring more details and the zones sufficiently flat are represented by a coarse resolution. This operation results in a procedure of subdivision and simplification during animation.

In order to carry out the principle of the multi-resolution, the subdivision scheme and the subdivision and the simplification criterion implemented in [12] are used. However, in this work, the subdivision scheme is implemented with an irregular mesh generated by the triangulation of Delaunay and the finite element method.

In order to avoid the divergence of the simulation a positions correction method of the particles is proposed

\subsection{Subdivision method (polyhedral scheme)}

In this step the polyhedral scheme [25] is chosen, this scheme preserves the original shape of the subdivided triangle. The polyhedral scheme consists in subdividing a triangle by adding new points in the middle of its edges and new edges connecting these points (figure 2).

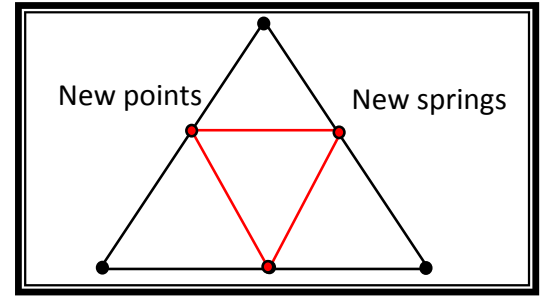

Figure :2 Polyhedral scheme

The subdivision criterion relates to the value of the angle between the normal of the two triangles having a common edge (see figure 3). Indeed, according to [11] if this angle exceeds a definite critical angle $\alpha$, a subdivision is applied. On each additional level, the critical angle increases for weighting the importance of the irregularities: the same angle between two small triangles is less than between two large triangles. We thus add $\beta$ to the critical angle for each additional level [11]

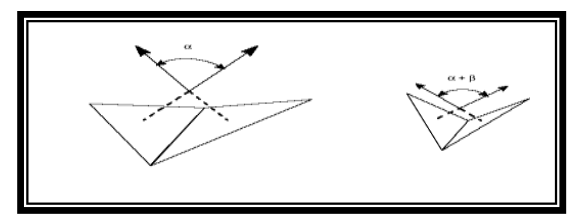

Figure:3 Augmentation of the critical angle when the triangle's surface decreases

The choice of $\alpha$ and $\beta$ depends on the degree of accuracy required. Moreover, the number of details' levels depends on the values selected on these angles and the initial form of mesh [12].

The principle of the subdivision can be summarized by the following algorithm:

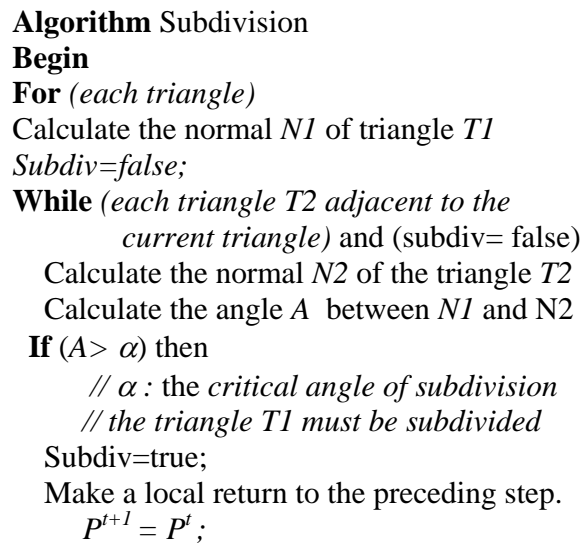

Subdivide the triangle (according to the polyhedral scheme)

Add a new point (particle) in the middle of each edge of the triangle

Connect the 3 new points to build a new triangle in the center of $\mathrm{T} 1$ and consequently three other triangles will be added (Figure 2)

Calculate the positions of the particles of the new triangles

Else

End if

Go to the next adjacent triangle.

End while

End for

End 


\subsection{Cancellation of a subdivision}

To optimize the simulation, the subdivision in the zones sufficiently flat is canceled. Thus, the flat areas are detected by calculating the angles between the normal of the subdivided triangles. If triangles in a refined zone are sufficiently flat, a local return to the preceding step is done. The particles forming part of this zone are given in the preceding state. And thus, the subdivision is canceled while recalculating the positions and speeds of the particles remaining in this area. This can be translated by the following algorithm:

\section{Algorithm Cancellation of subdivision \\ Begin}

For (each triangle)

If (the triangle $T$ is subdivided) then

$\mathrm{I}=0$;

Cancel=false;

Calculate the normal $N 1$ of the triangle of center $T 1$

For (each triangle T2 adjacent to the triangle T1)

Calculate the normal $N 2$ of the triangle $T 2$

Calculate the angle $A$ between $N 1$ and N2

If $((A>=0)$ and $(A<=\beta))$ then

$/ / \beta$ it's an angle sufficient small

$$
I=I+1 \text {; }
$$

End if

\section{End for}

If $(I=3)$ then

Cancel $=$ false ;

while (there is triangles T3 adjacent to the

$$
\text { triangle } T) \text { and }(\text { Cancel }=\text { false })
$$

Calculate the normal $N 3$ and $T 3$

Calculate the angle $A l$ between $N 1$ and N3

$$
\text { If }((A l>=0) \text { and }(A l<=b e t a)) \text { then }
$$

Cancel=true

\section{Else}

Go to the next triangle

\section{End if}

\section{Endwhile}

\section{If $($ cancel $=$ true $)$ then}

// the subdivision of triangle T must be cancelled

Make a local return to the preceding step.

$$
P^{t+1}=P^{t} \text {; }
$$

Cancel the subdivision

Remove triangles and particles added in subdivision step (figure 4)
Recalculate the position of the particles of triangle $T$.

\section{End if}

End if

End for

End

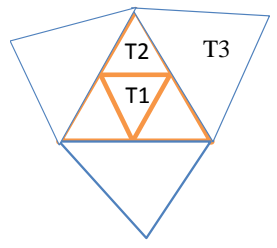

Before cancelling the subdivision

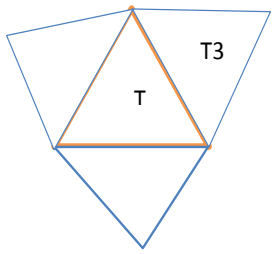

After canceling the subdivision

\section{Figure 4 Cancellation of the subdivision of the triangle $T$}

The cancellation of a subdivision is relatively complicated because it is always necessary to keep the original form of the mesh. And thus, the opposite direction of the subdivision polyhedral is chosen, i.e. if a triangle is already subdivided a comparison is done between the normal of the triangle of the center and the normal of each adjacent triangle which are created during the subdivision. Also a comparison between the normal of the triangle of the center and the normal of the adjacent triangles to the initial triangle (the subdivided triangle). If it is found that the surface occupied by the four triangles is sufficiently flat the subdivision is canceled, the new points as well as the new edges which were added during the subdivision are eliminated to obtain the initial triangle (see previous algorithm).

\section{POSITION CORRECTION}

The dynamic inverse procedure was used for the first time by Provot [26] because it allows correcting the super-elongation of the springs in the mass-spring system. Nevertheless, in this work the divergence of the simulation cannot be avoided with this method, since the values of the positions increase quickly, which always involves the increase in the forces and velocities and consequently the cloth tearing. The method proposed in this paper consist of correction of these values by limiting the movement of each particle, i.e. if the distance between two successive positions exceeds a certain value (threshold determined after several tests) the position of the particle must be corrected. The correction is thus to add half of the difference between the two successive positions of the considered particle to its previous position (its position at time $\mathrm{t}-1$ ), the correction must be applied several times depending on the size and shape of cloth, until the convergence of the model is assured.

The principle of this position correction method can be summarized in the following algorithm:

Algorithm Position correction

Begin

For (each particle)

For $\left(n b=1\right.$ to $\left.n \_i t\right) / / n$ it: number of iterations

Calculate the difference diff between the positions $P$ at time $t$, and $t+1$ of particles:

$$
\text { diff }=P^{t+1}-P^{t}
$$


Calculate the distance dist between the positions $P$ at time $t$ et $t+1$ of the particle :

$$
\text { dist= }=\text { diff } \mid \text {; }
$$

if (dist> threshold) then

Correct the current position:

$$
P^{t+1}=P^{t}+\operatorname{diff} / 2
$$

\section{End if}

\section{End for}

\section{If (dist> threshold) then}

Correct the velocity according the time- step $h$ :

$$
V^{t+1}=\left(P^{t+1}-P^{t}\right) / h ;
$$

\section{End if}

\section{End for}

End

This method has enabled us to reduce the error rate of displacement (see section 5).

\section{RESULTS AND DISCUSSION}

This section presents results of the multi-resolution cloth animation method. Simulations have been performed on the i5 Intel processor with 4 Go of RAM. Cloth and garment models used in this work were represented by an irregular triangular mesh generated by Fashionizer (which is a tool of design of virtual clothing, developed in the MIRALab Laboratory at the University of Geneva).

In the experimental tests, various pieces of cloth are used which having different sizes and resolutions. The results indicate that the computation time increases in an exponential way with the increase of the mesh resolution (see figure 5).

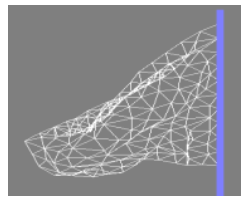

Cloth1: 302 triangles

$$
\mathrm{T}=5 \mathrm{sec} \text {. }
$$

Cloth2: 4874 triangles

$$
\mathrm{T}=75 \mathrm{sec} \text {. }
$$

Fig:5 Pieces of cloth animated with different sizes and resolutions (2500 iterations).

The examples below, illustrate garment animation of various types of cloth (linen, silk and cotton). Figures 6, 7 and 8, demonstrate that the garment movement depends on the type of cloth i.e the cloth physical properties. Indeed, the tests concerning animation without position correction (see figures $\mathrm{a}, \mathrm{c}$, and e) generated a divergence in the calculation of the forces applied to the particles. This induces, after a certain number of iterations, the divergence in the calculation of the particles' velocities and positions (case of garment with the linen in figure 6. a). This is imputable to the increase in the distance between the cloth's particles progressively of the animation process

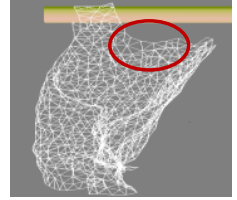

(a) $\mathrm{t}=13 \mathrm{~s}, 1130$ iterations without position correction.

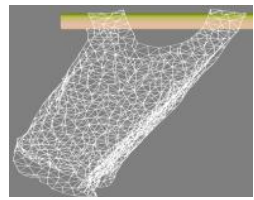

(b) $t=23 \mathrm{~s}, 2000$ iterations with position correction
Fig:6 Garment animation with Linen.

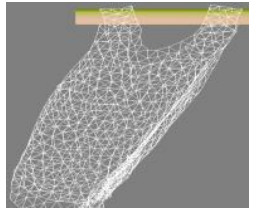

(c) $\mathrm{t}=22 \mathrm{~s}, 2000$ iterations without position correction.

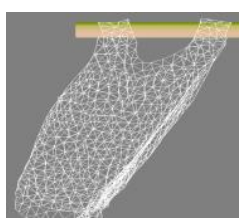

(d) $\mathrm{t}=23 \mathrm{~s}, 2000$ iterations with position correction.
Fig:7 Garment animation with silk.

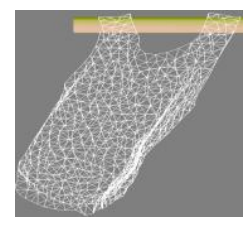

(e) $\mathrm{t}=23 \mathrm{~s}, 2000$ iterations without position correction.

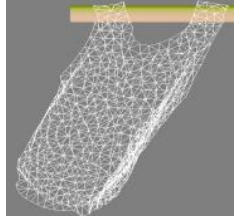

(f) $\mathrm{t}=23 \mathrm{~s}, 2000$ iterations with position correction.
Fig:8 Garment animation with cotton.

The following animation's tests relate to the integration of the multi-resolution in the model. By implementing the method of subdivision and simplification, it was necessary to correct the positions to ensure the convergence of the model. At this level, the problem was the criterion of subdivision, i.e. the choice of the angle $\alpha$ between the normal of the adjacent triangles to be subdivided. Table 1 below gives the tests performed with different angles.

Table 1 Variation of the number of subdivisions performed and cancelled and the number of triangles according to the subdivision angle (animation tests of a garment/Cylinder with 1000 iterations)

\begin{tabular}{|c|c|c|c|c|}
\hline Angle & $\begin{array}{c}\text { Number of } \\
\text { performed } \\
\text { subdivisions }\end{array}$ & $\begin{array}{c}\text { Number of } \\
\text { cancelled } \\
\text { subdivisions }\end{array}$ & $\begin{array}{c}\text { Number of } \\
\text { triangles }\end{array}$ & $\begin{array}{c}\text { Time } \\
\text { (sec.) }\end{array}$ \\
\hline $40^{\circ}$ & 69180 & 67491 & 8254 & 109 \\
\hline $50^{\circ}$ & 48256 & 46953 & 6710 & 72 \\
\hline $60^{\circ}$ & 33341 & 32332 & 5534 & 54 \\
\hline $65^{\circ}$ & 31591 & 30627 & 5354 & 51 \\
\hline $70^{\circ}$ & 26763 & 25903 & 4938 & 46 \\
\hline $75^{\circ}$ & 22480 & 21702 & 4610 & 41 \\
\hline $80^{\circ}$ & 18539 & 17807 & 4426 & 38 \\
\hline $90^{\circ}$ & 14672 & 14093 & 3814 & 32 \\
\hline $100^{\circ}$ & 11792 & 11336 & 3322 & 28 \\
\hline $120^{\circ}$ & 9861 & 9485 & 3002 & 25 \\
\hline $140^{\circ}$ & 6335 & 6095 & 2458 & 22 \\
\hline $160^{\circ}$ & 2820 & 2618 & 2306 & 20 \\
\hline
\end{tabular}

The results obtained in table 1 , show that the number of subdivisions performed and cancelled is inversely proportional to the considered angle $\alpha$.

Moreover, if the angle considered is small, any subdivision appears useful because in this case even the areas of cloth which are sufficiently flat will be subdivided. Also, if the value of the angle is too large, then the number of subdivisions decreases considerably. In this case, the 
subdivision is performed only if the area of the cloth is too wrinkled. For that a median value appears more interesting in the subdivision process (see the results grayed in table 1 above).

Table 1 above shows that any reduction in the angle $\alpha$ implies the increase in the number of triangles of the mesh and the computation time. The latter can also vary according to the number of levels of details used. Figure 9 illustrates the variation of the number of triangles according to the angle of the subdivision and the selected number of levels of details.

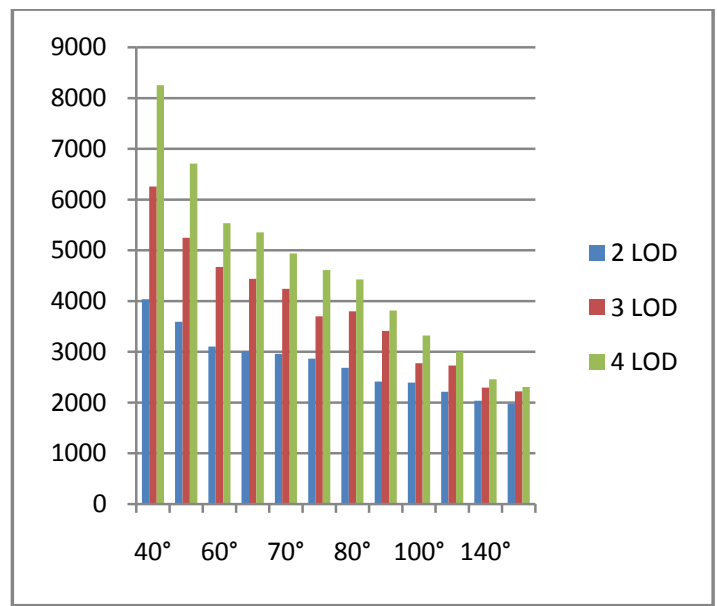

Fig: 9 The variation of the number of triangles according to the angle of subdivision and the selected number of levels of details.

Furthermore, figure 11 shows the animation of a piece of cloth and a garment. The two examples, cloth/sphere and garment/cylinder, enabled us to emphasize the form of cloth.

In the first case the number of triangles remains stable in the first iterations owing to the fact that the cloth is represented by a flat surface. This is not the case in the second example (garment/cylinder), the number of triangles increases in the first iterations owing to the fact that the cloth has a quite precise form at the beginning. This induces the variation of forces, speeds and positions from the beginning (see figure $10)$.

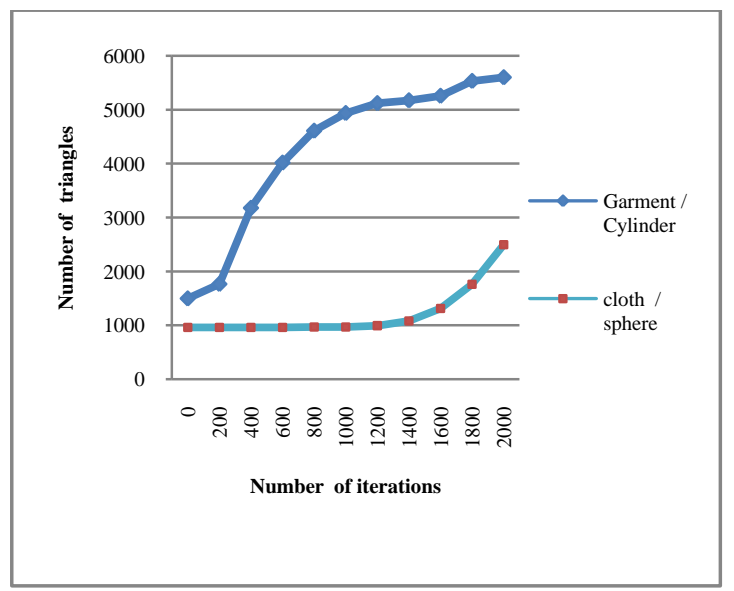

Fig:10 Variation of the number of triangles according to the number of iterations

The following figure shows three stages of the variation of the numbers of triangles during the simulation.

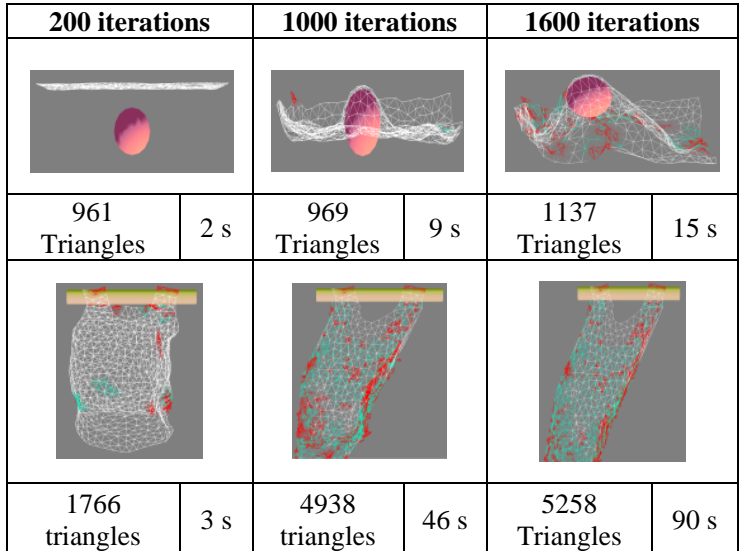

Fig: 11Stages of animation of a piece of cloth which fall on a sphere and garment (the subdivided triangles are in red and the subdivided and cancelled triangles are in green)

According to the experimental tests, the following conclusions can be drawn:

- The cloth movement depends on the physical properties of cloth. This is why it is difficult to fix the parameters of the external forces.

- Each piece of cloth and each form of garment require different parameters.

- In these simulations, a cotton cloth is used essentially in order to study the factors which influence the integration of the multi-resolution, namely, the subdivision criterion, i.e. the angle of subdivision alpha, the number of LODs and the form of the cloth.

\section{CONCLUSION}

In the context of cloth simulation, explicit integration methods used to determine the new particle positions always requires a very small time step to ensure the convergence of the model. This work proposes a method based on particle position correction, in which the convergence of the model can be ensured. Thus the displacement of each particle at each iteration is limited, so that each particle cannot move more than a certain distance (threshold) which it is determined after several tests. The multi-resolution cloth simulation is based on the first order finite element method. This model is expressed by a simple particle system, and then the multi-resolution is exploited. This method makes it possible to improve simulation while minimizing the computation time.

Therefore, the collision handling by the human body must be taken into account to increase the degree of realism.

\section{REFERENCES}

[1] N. Magnenat-Thalmann, P. Volino. "From early draping to haute couture models: 20 years of research". The Visual Computer 21: 506-519, 2005.

[2] K. Choi, H. Ko, "Research problems in clothing simulation". Computer Aided Design, 37(6): 585-592, 2005.

[3] M. Desbrun, P. Schröder and A. Barr, "Interactive animation of structured deformable objects", Proc. of Graphics Interface'99, Kingston, Ontario, Canada, 1999.

[4] M. Meyer, G. Debunne, M. Desbrun, and A. H. Barr. "Interactive animation of cloth-like objects in virtual 
reality. Journal of Visualization and Computer Animation", 12(1): 1-12, 2001.

[5] K. Choi and H. Ko. "Stable but responsive cloth". Proceedings of SIGGRAPH' 02, ACM Transactions on Graphics, 21(3): 604-611, San Antonio, Texas, USA, 2002.

[6] O. Etzmuss, M. Keckeisen and W Strasser, "A fast finite element solution for cloth modeling", Proceedings of the 11th Pacific Conference on Computer Graphics and Applications, PG '03, IEEE Computer Society: 244-251, Washington, DC, USA, 2003.

[7] P. Volino and N. Magnenat-Thalmann. "Implicit midpoint integration and adaptive damping for efficient cloth simulation". Computer Animation and Virtual Worlds, 16(3-4): 163-175, 2005.

[8] P. Volino and N. Magnenat-Thalmann. "Simple linear bending stiffness in particle systems". In Proceedings of the Eurographics/ACM SIGGRAPH Symposium on Computer Animation, Vienna, Austria, 2006.

[9] P. Volino, N. Magnenat Thalmann and F. Faure. "A simple approach to nonlinear tensile stiffness for accurate cloth simulation”, ACM Trans. Graph. 28(4): $1-105,2009$.

[10] D. Hutchinson, M. Preston and T. Hewitt, "Adaptive refinement for Mass/Spring Simulations", Proceedings of Seventh Eurographics Workshop on Animation and Simulation, Poitier, France, 1996.

[11] P.-Y. Burgy. "Intégration de la multirésolution dans un système de déformation masse-ressort". Federal Polytechnic school of Lauranne, 2000.

[12] S. Benameur and N. Djedi, "Intégration de la multirésolution dans un système masse-ressort : Application à l'animation de tissu", TAIMA'05, Tunisia, 495-500, 2005.

[13] J. Villard and H. Borouchaki. "Adaptive meshing for cloth animation", Engineering with Computers, No 20: 333-341, 2005.

[14] F. Birra and M. Santos, "Towards Efficiency in Cloth Simulation”, AMDO 2008, LNCS 5098: 144-155, 2008.
[15] Y. Lee, S. Yoon, S. Oh, D. Kim and S. Choi, "Multiresolution cloth simulation". Computer Graphics Forum (Pacific Graphics) 29(7): 2225-2232, 2010.

[16] L. Li and V. Volkov, "Cloth Animation with Adaptively Refined Meshes", 28th Australasian Computer Science Conference, Vol. 38: 107-113, 2005.

[17] D. Baraff and A. Witkin, "Large steps in cloth simulation”, SIGGRAPH Computer Graphics, 43-54, 1998.

[18] T. Le Thanh and A. Gagalowicz, "Fast Virtual Cloth Energy Minimization”, MIRAGE 2007, LNCS 4418, 139-149, 2007.

[19] O. Etzmuss, J. Gross and W Strasser, "Deriving a particle system from continuum mechanics for the animation of deformable objects", IEEE Transactions on Visualization and Computer Graphics 9, 4, 538-550, Oct. 2003.

[20] T. Brochu, E. Edwards and R. Bridson, "Efficient geometrically exact continuous collision detection", ACM Trans. Graph. 31 (4):1-96, 2012.

[21] J. Bender and C. Deul, "Efficient cloth simulation using an adaptive finite element method", Virtual Reality Interactions and Physical Simulations (VRIPhys), Darmstadt, 2012

[22] Jan Bendera, Daniel Weberb, Raphael Diziol, "Fast and stable cloth simulation based on multi-resolution shape matching", Computers \& Graphics, volume 37(8): 945954, 2013.

[23] E-H. Taibi, "Caractérisation, modélisation et simulation $d u$ comportement d'un tissu textile", Ph.D. thesis, Mohamed V university Morocco \& University of Bordeaux I, France, 2001.

[24] P. Volino, F. Cordier and N. Magnenat Thalmann, "From early virtual garment simulation to interactive fashion design", Computer-Aided Design, 37: 593-608, 2005.

[25] B. Sharp, "Subdivision Surface Theory", Game Developer, Vol. 7(1): 34-42, 2000.

[26] X. Provot, "Deformation constraints in a mass-spring model to describe rigid cloth behaviour". Graphics Interface:147-154, 1995. 